\title{
Sequential Observation of Liver Cell Regeneration after Massive Hepatic Necrosis in Auxiliary Partial Orthotopic Liver Transplantation
}

\author{
Miri Fujita, M.D., Hiroyuki Furukawa M.D., Masahiro Hattori, M.D., Satoru Todo, M.D., \\ Yusuke Ishida, M.D., Kazuo Nagashima, M.D. \\ Departments of Surgical Pathology and Surgery, Hokkaido University Hospital, and Laboratory of \\ Molecular \& Cellular Pathology, Hokkaido University School of Medicine (MF, HF, MH, ST, YI, KN), \\ Sapporo, and Core Research for Evolutional Science and Technology, Japan Science and Technology \\ Corporation (KN), Tokyo, Japan
}

The morphogenesis of hepatocytes after massive hepatic necrosis to recovery through liver cell regeneration has not been fully understood. Sequential biopsies were performed on the native liver of a 22-year-old man who underwent auxiliary partial orthotopic liver transplantation 1 month after fulminant hepatitis. Auxiliary partial orthotopic liver transplantation was successful, and the biopsy samples permitted us to examine the regenerating process of hepatocytes after massive necrosis. At the time of auxiliary partial orthotopic liver transplantation (postoperative day 0 ), $95 \%$ of hepatocytes were lost and a few ductules were found in the portal areas. The ductules stained with cytokeratin 19. At postoperative day 7 , the ductules began to increase in size and number and became dilated over a period of 1 month, when individual hepatocytes with clear cytoplasm appeared from the ductules. As the differentiation of hepatocytes increased, the expression of cytokeratin 19 was found to decrease. From 2 to 3 months, all of the ductules were transformed into hepatocytes, and they began to form round cell clusters. From 3 to 6 months, the round cell clusters became organized into trabecula with fibrosis. From 6 to 12 months, a lobular architecture was established, and by 14 months, the necrotic liver was fully recovered to normal. This study by examination of sequential biopsies demonstrates the progression of the regenerating process from total hepatic necrosis to complete recovery.

Copyright () 2000 by The United States and Canadian Academy of Pathology, Inc.

VOL. 13, NO. 2, P. 152, 2000 Printed in the U.S.A.

Date of acceptance: August 30, 1999.

Address reprint requests to: Kazuo Nagashima, M.D., Laboratory of Molecular \& Cellular Pathology, Hokkaido University School of Medicine, Kita 15, Nishi 7, Kita-ku, Sapporo 060-8638, Japan; e-mail: knagasi@ med.hokudai.ac.jp; fax: 81-11-706-7806.
KEY WORDS: Auxiliary partial orthotopic liver transplantation, Ductular hepatocytes, Fulminant hepatitis, Liver cell regeneration.

Mod Pathol 2000;13(2):152-157

Fulminant hepatitis is an uncommon complication of acute viral hepatitis and is seen in fewer than $1 \%$ of cases of acute viral hepatitis, type A and type B. This carries a poor prognosis with a mortality rate of more than $50 \%$ in most series (1). Auxiliary partial orthotopic liver transplantation (APOLT) has recently been performed in patients with acute liver failure and who developed neurologic complications such as fulminant hepatitis accompanied by hepatic coma (2-7) and in patients with noncirrhotic metabolic diseases such as ornithine carbamoyltransferase deficiency (8). In the former, especially in young patients, recovery of liver function and full regeneration of liver cells of the native liver, as well as the eventual withdrawal of immunosuppression, can be expected (6). Auxiliary heterotopic liver transplantation for fulminant hepatitis has antedated APOLT $(9,10)$. In comparison with auxiliary heterotopic liver transplantation, APOLT has some physiologic advantages, such as those relating to portal blood flow, abdominal tissue perfusion, and venous return to the heart.

Liver cell regeneration after massive hepatic necrosis as a result of fulminant hepatitis has not been pathologically examined in detail because most patients have had a fatal course; even if they had survived, biopsy examination often would have been life threatening because of hemorrhagic diathesis. Recently, however, it has been shown that liver cell regeneration after necrosis can be observed in the native liver after APOLT $(2,5-7)$, and there have been a limited number of reports describing regenerative liver cells in fulminant hepatitis in APOLT. In these reports, ductular hepato- 
cytes were considered to be the putative progenitor cells of hepatocytes (11-14). However, these findings have been based on a few biopsy studies, and sequential histologic observations have not been carried out to understand fully liver cell regeneration.

We experienced a young adult case of APOLT for fulminant hepatitis with hepatic coma as a result of hepatitis type B viral infection. Coexistence of grafted liver and native liver was monitored clinically and histologically for 14 months. This provided an opportunity to examine the origin and processes of liver cell regeneration and specifically to define the role of ductular hepatocytes.

\section{CASE REPORT}

A 22-year old man complained of loss of appetite on August 3, 1997. He visited a doctor on August 6, and his liver function tests disclosed abnormal values of GOT $1950 \mathrm{IU} / 1$ and GPT $2344 \mathrm{IU} / 1$. He was admitted to the Teine Keijinkai Hospital in Sapporo City, Japan. The values of GOT and GPT were noted to be further elevated: GOT $2145 \mathrm{IU} / \mathrm{l}$ and GPT 2615 IU/l. Because conservative therapy did not yield a response, consecutive plasma exchanges were performed seven times. However, he fell into a hepatic coma, Grade 4, which necessitated respiratory management with a mechanical respirator. Cooling therapy of body temperature was introduced to reduce intracranial pressure on September 2. The following morning, the patient was transferred to Hokkaido University Hospital for possible liver transplantation from his father, age 54.

Laboratory data on admission was as follows: total bilirubin $14.7 \mathrm{mg} / \mathrm{dl}$, GOT $149 \mathrm{IU} / \mathrm{l}$, GPT 147 IU/l, NH3 $209 \mathrm{mg} / \mathrm{l}$. Serological tests of hepatitis type B infection were positive for HBs-Ag, HBs-Ab, $\mathrm{HBe}-\mathrm{Ab}$, and $\mathrm{HBc}-\mathrm{Ab}$ but negative for $\mathrm{HBe}-\mathrm{Ag}$.

APOLT was selected by the patient's father for rescue therapy and was carried out after we received informed consent from the patient's parents and approval of the Ethics Committee of Hokkaido University. Steatosis of the donor liver was suggested by echogram, and an intraoperative frozensection diagnosis was performed for evaluation of the donor liver. Although mixed vesicular steatosis was observed in up to $30 \%$ of the liver, the liver was used for APOLT because the type of steatosis was not macrovesicular and not of a severe grade.

The procedure of transplant surgery (on September 4) was as described elsewhere (3). The left lobe of the liver explanted from the recipient's liver weighed $168 \mathrm{~g}$ and showed massive parenchymal necrosis up to $95 \%$, and only $5 \%$ of the liver revealed macroscopic regenerative nodules. The engrafted left lobe weighed $600 \mathrm{~g}$. After APOLT, both grafted liver (left lobe) and native liver (right lobe) were monitored by sequential needle biopsies. Acute rejection of mild to moderate grade was detected on the section from the grafted left lobe on the 20th day after transplantation. Intensive immunosuppressive therapy caused transient cytomegalovirus antigenemia thereafter for a week. However, successful treatment of rejection and cytomegalovirus infection resulted in discharge of the patient on October 15, 1997.

The change of size in the native and grafted livers was evaluated by computed tomographic imaging at 1 month, 3 months, and 9 months after APOLT. The estimated native and grafted liver weights were as follows: $380 \mathrm{~g}$ and $600 \mathrm{~g}$ at 1 month, $390 \mathrm{~g}$ and $560 \mathrm{~g}$ at 3 months, and $440 \mathrm{~g}$ and $700 \mathrm{~g}$ at 9 months. The prothrombin time was 16 seconds on the transferred day and improved to 13 seconds at postoperative day (POD) 14. The values of prothrombin time after discharge were between 11 and 12 seconds through the entire course. Viral serology of HBs-Ag and HBe-Ab were converted to negative soon after the transferred day and at 6 months after APOLT, respectively. Positive HBs-Ab and $\mathrm{HBc}-\mathrm{Ab}$ and negative HBe-Ag continued through the course.

\section{MATERIALS AND METHODS}

Sequential liver biopsy materials were taken with a 16-gauge needle on POD 7, 16, 20, 29, and 34, and two open and wedge biopsies were performed on the date of transplant surgery (POD 0) and on POD 10. Native liver biopsies were included in the materials of POD $0,7,10$, and 29; after discharge, they were added at 2, 3, 4, 6, 9, 10, 12, and 14 months after APOLT, combined with graft liver biopsies to monitor acute rejection (Fig. 1).

A total of 12 biopsy materials of native liver (pretransplant left lobe at POD 0; right lobe thereafter) and 15 biopsies of grafted liver (left lobe) of the patient were processed by formalin-fixed, paraffinembedded sections using standard methods. They were stained with hematoxylin-eosin, Masson's trichrome, and reticulin fiber staining and were observed microscopically. Immunohistochemistry was performed on the paraffin sections from native liver biopsies to detect the specific markers of liver cell regeneration. Antibodies used were monoclonal antibodies for cytokeratin (CK) 14, CK18, CK19, (Novocastra, Newcastle upon Tyne, UK) and CAM 5.2 (Becton Dickinson, Mountain View, CA) and a polyclonal antibody for alpha-1 antitrypsin (DAKO, Glostrup, Denmark). Monoclonal antibodies for hepatitis B viral surface and core antigens (HBs-Ag and $\mathrm{HBc}-\mathrm{Ag}$ ) were also used to detect virus-infected cells. Antisera dilution, antigen retrieval methods, 


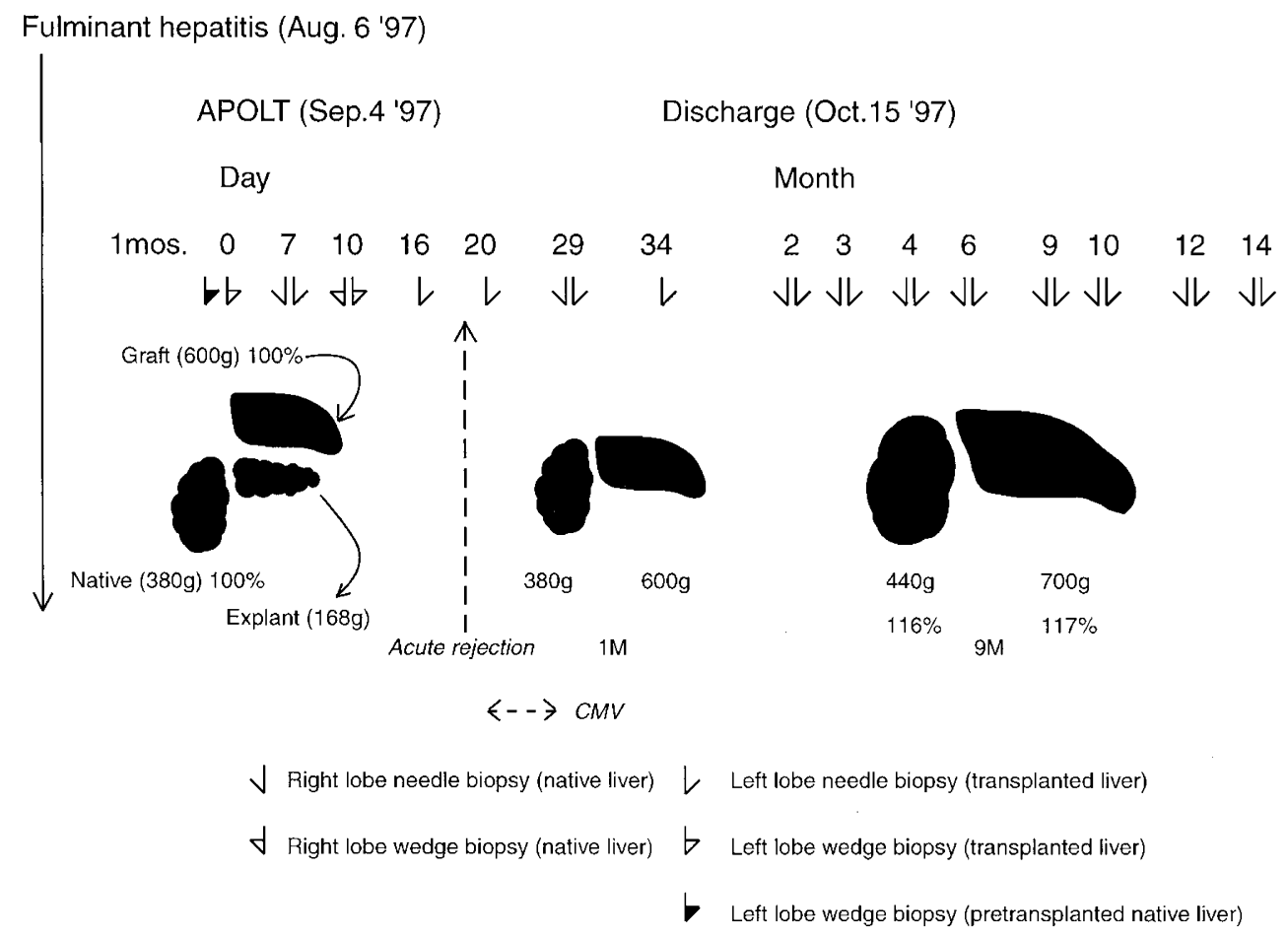

FIGURE 1. Clinical course and pathologic examination.

and other technical procedures were used according to the manufacturer's recommendations.

\section{RESULTS}

The histology of the native liver showed massive hepatic necrosis, but ductules were observed in the periportal and portal areas on the day of APOLT (Fig. 2A), approximately 1 month after the onset of fulminant hepatitis. At this time, ductules were clearly demonstrated by CK19 staining (Fig. 2B). The ductules were irregular in shape and clustered in the portal areas. Successful APOLT revealed increased ductules with dilated lumen and enlarged epithelial cells on POD 7. The ductules primarily consisted of basophilic cells with high cytoplasmic nuclear ratio, but they were arranged irregularly without forming a line-up and apical basal orientation (Fig. 2C). CK19 staining labeled the tubules strongly (Fig. 2D). We considered the gradual transformation of the ductules to hepatocytes to be an initial stage of hepatocyte regeneration. Centrilobular areas showed still necrosis with hemorrhagic changes. Open and wedge biopsy on POD 10 disclosed the development of collagen fiber in the massive necrotic regions, especially in the centrilobular areas. The ductules in the portal areas were increased in number and were composed of enlarged cells with dilated lumina. On POD 29, ductular proliferation became prominent (Fig. 3A) and a single hepatocyte was observed among the ductular epithelium of the native liver (Fig. 3B). As the hepa-
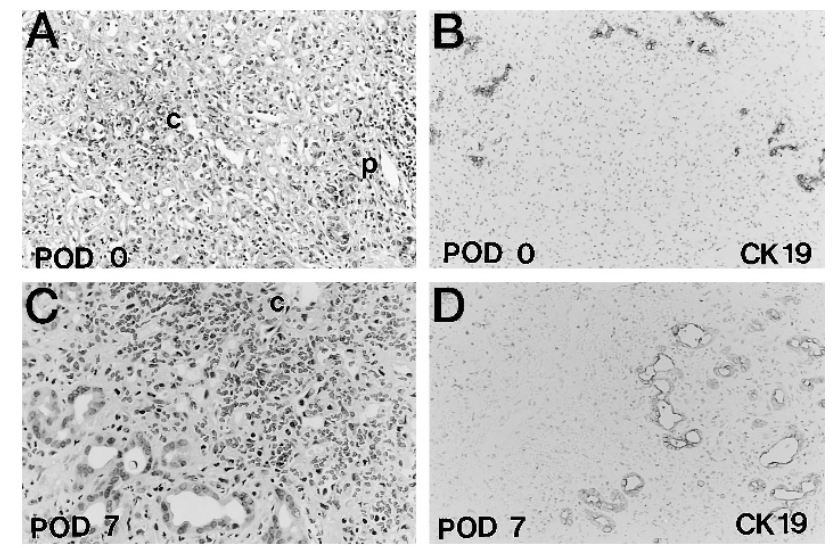

FIGURE 2. Massive hepatic necrosis, and ductular cells from postoperative day (POD) 0 to POD 7 after auxiliary partial orthotopic liver transplantation. A, intraoperative (POD 0) wedge biopsy of the native liver showed massive hepatic necrosis and periportal ductular structure $(\mathrm{HE}, 200 \times)$. B, ductules were found in the probable portal areas and were intensely labeled with cytokeratin (CK) 19

(immunostain, $140 \times$ ). C, irregular dilatation of ductules was seen at POD 7. The ductules consisted of slightly basophilic cells. Note the hemorrhagic necrosis around the central vein $(\mathrm{HE}, 330 \times)$. D, proliferation of ductules at POD 7 was clearly demonstrated by CK19 positive ductules (immunostain, $200 \times$ ). c, centrilobular area; p, portal area.

tocytes developed, they were large and oval in shape and had clear cytoplasm. The ductules were still intensely labeled with CK19, although the differentiated hepatocytes had only faint staining with CK19 (Fig. 3C). The centrilobular areas were still necrotic, but dilatation of central veins and sinusoids developed.

Two months after APOLT, the necrosis disappeared, ductules decreased in number, and numer- 

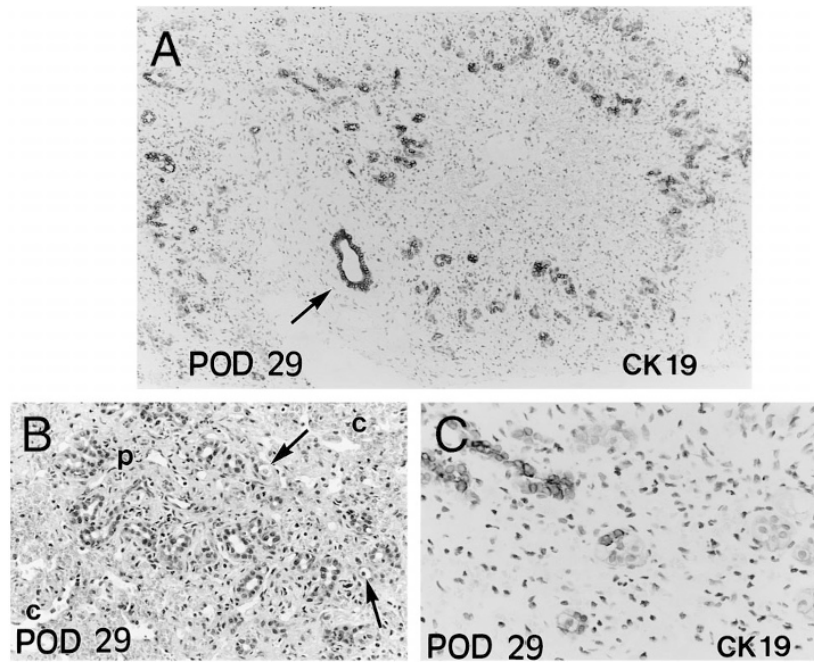

FIGURE 3. Ductular proliferation and initial appearance of a hepatocyte at postoperative day (POD) 29. A, increased number of periportal ductules was seen at POD 29. They were well demonstrated by cytokeratin (CK) 19 staining. Note the CK19-positive bile duct (arrow) (immunostain, $100 \times$ ). B, individual hepatocytes were first seen around the ductules (arrows). They were round in shape and had clear cytoplasm (HE, 265×). C, CK19 showed faint staining of the hepatocytes in contrast to intense staining of ductules (immunostain, $400 \times)$. c, centrilobular area; p, portal area.

ous clear individual hepatocytes appeared in the native liver (Fig. 4A). Hepatocytic differentiation from ductules became abundant, and CK19positive ductules decreased (Fig. 4B). At 3 months after APOLT, regenerative liver cells formed round clusters without trabecular pattern but partly showed an acinar pattern that was different from normal liver cords (Fig. 4C). The ductules were markedly decreased in number, and the more differentiated the hepatocytes became, the less intense was the staining with CK19 (Fig. 4D).

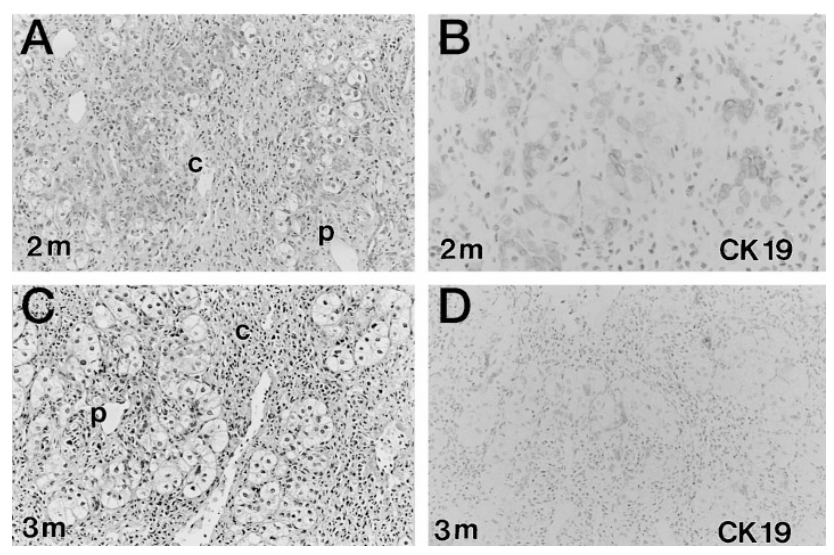

FIGURE 4. Increased hepatocytes at 2 and 3 months after surgery. A, ductular hepatocytes were transformed into round and clear hepatocytes mostly near the portal areas (p) (HE, 200×). B, decrease of ductules were clearly seen by cytokeratin (CK) 19 with a decreased expression of CK19 in newly formed hepatocytes (immunostaining, $400 \times)$. C, hepatocytes increased in number and became clustered, forming a round ball or an acinar pattern between central vein (c) and portal areas (p) $(200 \times)$. D, ductules that expressed CK19 disappeared, leaving a few trace positive cells (immunostaining, 200×).
Three, 4, and 6 months after APOLT, the number of hepatocytes increased and became arranged in a trabecular pattern (Fig. 5A). Ductules disappeared, and only the bile canaliculi were observed in the portal area. At 6 months after APOLT, fibrosis was seen around the hepatic cord (Fig. 5B). By 1 year after APOLT, hepatic lobules were established and fibrosis had disappeared gradually (Fig. 5C). Finally, nearly $80 \%$ of the biopsied liver tissue showed complete regeneration without differences from normal liver tissue at 1 year and 2 months after APOLT (Fig. 5D). Obviously differentiated hepatocytes were negative for CK19, and none of the liver tissue was labeled with CK14. In comparison with CK19, both CK18 and CAM 5.2 labeled both ductules and hepatocytes through the course. Alpha-1 antitrypsin labeled only a part of ductules and hepatocytes, and plasma in the vessels. Only one biopsy from the grafted liver at POD 20 revealed focal acute rejection with central venulitis. Antisera to hepatitis B virus-related antigens HBs-Ag and HBc-Ag did not label the hepatocytes of either native or grafted livers at any time through the entire course.

\section{DISCUSSION}

The first successful case of APOLT was reported by Gubernatis et al. (2) in 1991 in a 33-year-old woman who had fulminant hepatic failure as a result of viral hepatitis, type A. Histologically, the native liver showed $80 \%$ destruction of the hepatic parenchyma followed by its complete restoration approximately 5 months after APOLT. The clinical status of the patient over the period of 16 months after transplantation was excellent, and immunosuppressive therapy was tapered off to surrender

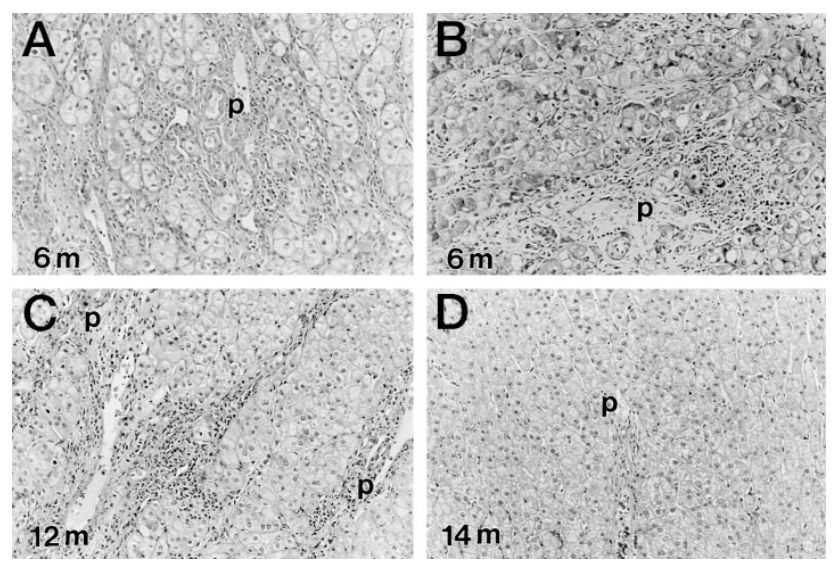

FIGURE 5. Hepatic lobe reorganization from 6 months to 14 months after surgery. A, clustered hepatocytes became organized in a trabecular pattern at 6 months $(\mathrm{HE}, 200 \times)$. B, fibrosis was seen around the hepatic trabeculae at 6 months (Masson trichrome stain, $200 \times$ ). C, hepatic lobules were established at 12 months. Perilobular fibrosis was still observed $(\mathrm{HE}, 200 \times$ ). D, hepatic lobules without fibrosis was seen at 14 months, which showed complete recovery to that of a normal liver $(\mathrm{HE}, 200 \times)$. p, portal area. 
the graft. Subsequently, Oldhafer et al. (3) described four cases of APOLT, one of which was the aforementioned case for acute liver failure, and all patients were alive. In this report, the previously described patient had a laparotomy for the repair of abdominal hernia 3 years after APOLT, and the surgeons observed an almost complete absorption of the graft at the time of surgery. The patient was in excellent condition without immunosuppressive therapy, and the remnant of the graft remained in situ. Two of the other three cases showed a quick recovery of the native liver, and focal necrosis of the graft was evident on imaging by cholescintigraphy and sonography. As a result, the grafts were removed 15 and 40 days after APOLT, respectively, and immunosuppression was withdrawn. The fourth case was complicated by arterial thrombosis after APOLT and resulted in a subsequent wholeliver transplantation. The patient recovered slowly after the second transplantation because of rejection and viral pneumonia. In this report, there were no histologic descriptions with respect to liver cell regeneration.

Boudjema et al. (4) and Bismuth et al. (5) reported series of APOLT performed in eight patients and five patients with fulminant and subfulminant hepatic failure, respectively. More than half of the patients showed full regeneration of liver cells in their native livers in both reports, and as a result, four and two of the grafts in each report were removed after tapering or stopping immunosuppressive therapy.

In terms of large-scale trials, a multicenter European study of auxiliary liver transplantation was reported by Chenard-Neu et al. (6). This study included 30 patients with fulminant hepatic failure, 24 of whom underwent APOLT. In six patients, the auxiliary graft was implanted heterotopically below the native liver. The overall survival rate was $63 \%$ after a 3- to 67-month follow-up, superior to the 1-year survival rate of $60 \%$ noted after orthotopic liver transplantation. Most important, 13 of the 19 survivors (68\%), which was $43 \%$ of the total 30 patients, had full recovery of the native liver and had stopped immunosuppressive therapy permanently. Twenty-two patients were histologically examined by biopsy for the evaluation of the native liver between two and nine times. Complete regeneration occurred in $78 \%$ of patients who were younger than 40 years, whereas fibrosis or cirrhosis occurred in $75 \%$ of those who were older than 40 years. In patients who had full hepatocyte regeneration in the native liver, the cause of acute liver failure was either viral hepatitis or acetaminophen overdose in $82 \%$, and the delay between the onset of jaundice and encephalopathy was less than 7 days in $87 \%$. These three criteria-age younger than 40 years, presence of viral hepatitis or acetaminophen overdose, and hyperacute course-all are indicators associated with a relatively favorable outcome in patients with acute liver failure. Our case fulfilled two of the three criteria: age younger than 40 years and hepatitis B virus infection.

Pereira et al. (7) proposed on the basis of the observations of seven cases of young patients with acute liver failure that having a high proportion of surviving hepatocytes within necrotic parenchyma was not a prerequisite for native liver regeneration, because in two cases of complete hepatocyte regeneration, there was $60 \%$ and $80 \%$ liver cell necrosis of the resected native liver, respectively. In addition, the authors suggested that macroscopic regenerative nodules within a small necrotic native liver seemed to be a predictor of the development of fibrosis or cirrhosis. Unlike their experience, our patient recovered without fibrosis or cirrhosis, although his liver showed not only macroscopic regenerative nodules in massive hepatic necrosis of the explanted small native left lobe but also fibrosis intermixed with remarkably regenerative hepatocytes in needle biopsies by 6 months after APOLT.

In the course of liver cell regeneration from massive hepatic necrosis, ductular proliferation plays an important role. Generally, two major types of ductular proliferation, also known as ductular reactions, are recognized: that seen in massive or submassive hepatic necrosis and that seen in mechanical biliary obstruction. The ductules in the former type have been called ductular hepatocytes because of suggestions that they are the putative progenitor cells of hepatocytes, and those of the latter type are called bile ductules and are thought to have characteristics of mature bile duct epithelium and are not related to hepatocyte regeneration (11-14). After the ductular proliferation was observed in our patient, the ductular epithelial cells that developed increased nuclear and cytoplasmic sizes, causing dilatation of the ductules. These ductular hepatocytes could be immunohistochemically labeled with CK19 during the ongoing regeneration of liver cells from ductules; these cells then had decreased and subsequently lost CK19 expression after complete differentiation to hepatocytes. This resembles the phenomenon in the fetal developing liver, where hepatoblasts expressed CK19 from 9 to 16 weeks' gestation, and subsequently fetal hepatocytes around 10 weeks' gestation lost the expression of CK19 (11). CK14 staining using monoclonal antibody was absolutely negative in our patient. Positive staining of ductular hepatocytes and bile ducts was obtained by using polyclonal antibody (13). In addition to the CK19 and CK14, CK7 and alpha fetoprotein are thought to label progenitor cells in the fetal liver. However, CK7 stain in diseased livers was performed on the frozen sections (15), and 
$\alpha$-fetoprotein staining was negative on the ductular hepatocytes in massive or submassive hepatic necrosis $(11,12)$. Further studies including in the fetal liver, various diseased livers, various tissues, or staining conditions are needed to clarify the expression of liver progenitor cell markers.

Our study revealed that ductular hepatocytes play the role of progenitor cells in the liver cell regeneration from massive hepatic necrosis after APOLT. The possible origin of ductular hepatocytes includes periportal injured hepatocytes, progenitor cells located in or near the canals of Herring, and a few stem cells in the portal and periportal areas persistent in the adult liver (11-13). Progenitor cells and stem cells are the most likely origin because of their site of appearance and the bipotential histologic and immunohistochemical characteristics of the bile duct epithelium and hepatocytes as in our patient.

In summary, our sequential histologic observations revealed that liver cell regeneration from massive hepatic necrosis started from proliferation of ductular hepatocytes at 7 to 10 days after APOLT, and hepatocyte differentiation in the ductular hepatocytes occurred at nearly 1 month after APOLT. CK19-positive ductular hepatocytes decreased in number as did the intensity of CK19 expression with the progression of liver cell regeneration 2 to 14 months after APOLT. As our case was massive hepatic necrosis as a result of fulminant viral hepatitis B infection, it would be of interest to know whether that arising from other causes might have a similar regenerative course.

Acknowledgment: The technical expertise of Mr. T. Endo, Mr. M. Arakawa, Mr. M. Shimizu, Mr. J. Moriya, Ms. Y. Shibuta, Ms. T. Kurokawa, and Ms. A. Funahashi is greatly acknowledged, and we thank them for their assistance.

\section{REFERENCES}

1. Lee RG. Acute hepatitis. In: Terasaki PI, Cecka JM, editors. Diagnostic liver pathology. St. Louis: Mosby; 1994. pp. 23-56.

2. Gubernatis G, Pichlmayr R, Kemnitz J, Gratz K. Auxiliary partial orthotopic liver transplantation (APOLT) for fulminant hepatic failure: first successful case report. World J Surg 1991;15:660-6.

3. Oldhafer KJ, Gubernatis G, Schlitt HJ, Rodeck B, Böker K, Pichlmayr R. Auxiliary partial orthotopic liver transplantation for acute liver failure: the Hannover experience. In: Lee RG. Clinical transplants. Los Angeles: UCLA Tissue Typing Laboratory; 1994. pp. 181-7.

4. Boudjema K, Cherqui D, Jaeck D, Chenard-Neu MP, Steib A, Freis G, et al. Auxiliary liver transplantation for fulminant and subfulminant hepatic failure. Transplantation 1995;59: 218-23.

5. Bismuth H, Azoulay D, Samuel D, Reynes M, Grimon G, Majno P, et al. Auxiliary partial orthotopic liver transplantation for fulminant hepatitis: the Paul Brousse experience. Ann Surg 1996;224:712-26.

6. Chenard-Neu MP, Boudjema K, Bernuau J, Degott C, Belghiti J, Cherqui D, et al. Auxiliary liver transplantation: regeneration of the native liver and outcome in 30 patients with fulminant hepatic failure-a multicenter European study. Hepatology 1996;23:1119-27.

7. Pereira SP, McCarthy M, Ellis AJ, Wendon J, Portmann B, Rela M, et al. Auxiliary partial orthotopic liver transplantation for acute liver failure. J Hepatol 1997;26:1010-7.

8. Uemoto S, Yabe S, Inomata Y, Nishizawa H, Asonuma K, Egawa $\mathrm{H}$, et al. Coexistence of a graft with the preserved native liver in auxiliary partial orthotopic liver transplantation from a living donor for ornithine transcarbamylase deficiency. Transplantation 1997;63:1026-8.

9. Moritz MJ, Jarrell BE, Armenti V, Radomski J, Carabasi RA, Zeitoun G, et al. Heterotopic liver transplantation for fulminant hepatic failure-a bridge to recovery. Transplantation 1990;50:524-6.

10. Metselaar HJ, Hesselink EJ, de Rave SD, ten Kate FJWT, Lameris JS, Groenland THN, et al. Recovery of failing liver after auxiliary heterotopic transplantation. Lancet 1990;335: $1156-7$

11. Rubin EM, Martin AA, Thung SN, Gerber MA. Morphometric and immunohistochemical characterization of human liver regeneration. Am J Pathol 1995;147:397-404.

12. Demetris AJ, Seaberg EC, Wennerberg A, Ionellie J, Michalopoulos G. Ductular reaction after submassive necrosis in humans: special emphasis on analysis of ductular hepatocytes. Am J Pathol 1996;149:439-48.

13. Haque S, Haruna Y, Saito K, Nalesnik MA, Atillasoy E, Thung $\mathrm{SN}$, et al. Identification of bipotential progenitor cells in human liver regeneration. Lab Invest 1996;75:699-705.

14. Fiel MI, Antonio LB, Nalesnik MA, Thung SN, Gerber MA. Characterization of ductular hepatocytes in primary liver allograft failure. Mod Pathol 1997;10:348-53.

15. Roskams T, de Vos R, Desmet V. "Undifferentiated progenitor cells" in focal nodular hyperplasia of the liver. Histopathology 1996;28:291-9. 